\title{
The implant-supported milled bar overdenture: A literature review
}

\author{
Antonio Bueno-Samper ${ }^{1}$, Manuel Hernadez-Aliaga ${ }^{2}$, José-Luis Calvo-Guirado ${ }^{3}$ \\ ${ }^{1}$ Dentistry Graduate. School of Dentistry. University of Murcia. Spain \\ ${ }^{2}$ Associate Professor of Clinical Adult Odontology. School of Dentistry. University of Murcia. Spain \\ ${ }^{3}$ Lecturer and Director of Implant dentistry Course. School of Dentistry,.University of Murcia. Spain
}

Correspondence:

C/ Antonio Machado, $121-2^{\circ} \mathrm{b}$.

Postcode: 03180

Torrevieja, Spain.

clinicadentalantoniobueno@tvt.es

Received: 18/11/2008 Accepted: 02/08/2009

\author{
Bueno-Samper A, Hernández-Aliaga M, Calvo-Guirado JL. The implant- \\ supported milled bar overdenture: A literature review. Med Oral Patol \\ Oral Cir Bucal. 2010 Mar 1;15 (2):e375-8. \\ http://www.medicinaoral.com/medoralfree01/v15i2/medoralv15i2p375.pdf

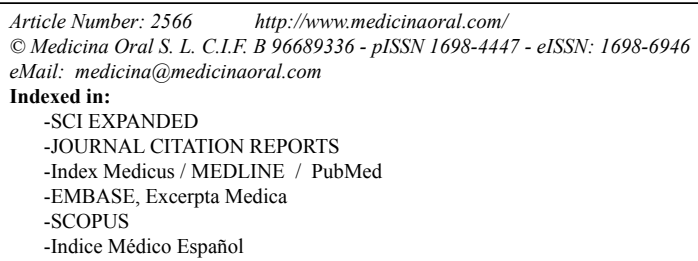

\begin{abstract}
Purpose: This bibliographical review aims to determine the present situation of the implant-supported milled bar overdenture as a therapeutic option in implantology, as well as to set and compare the techniques and materials currently used for making them. Materials and methods: By searching the main electronic bibliographical data in indexed Medline articles, we obtained a total number of 20 articles that fulfilled the requirements of this bibliographical review. Results: The implant-supported milled bar overdenture offers excellent long-term successful results, although complication rates are higher in emergency cases of patients initially planned for a fixed prosthesis. Krenmair and collaborators found cumulative survival rates of implants on milled bars after 5 years, of $99 \%$ in mandible cases, and of $97.8 \%$ in maxilla cases. No detailed protocols specifying the necessary number of implants or their characteristics have been found, though they are never made with less than 4 implants in mandible or 4-6 in the maxilla. Milled bars are screwed, generally cast in a precious metal alloy, with a metal or acrylic suprastructure and include attachments that regulate the tightness. The suprastructure adjusts precisely and rigidly to the milled bar, and presents similar biomechanical movements to those of fixed prostheses. The overdenture is removable and its prophylaxis is simple. Conclusions: The implant supported milled bar overdenture is a very interesting option in the treatment of patients with moderate to severe reabsorbed maxilla problems. It offers both the advantages of removable prostheses as well as the stability and retention of a fixed prosthesis.
\end{abstract}

Key words: Milled bar, implant overdentures, implant-supported overdentures.

\section{Introduction}

Osseous integrated dental implants have been proved successful in total and partial edentulous treatment cases (1-6). There is a great variety of alternative therapeutic prostheses at hand which, for practical reasons, will be classified as follows: Wholly or partially made of porcelain fixed prostheses, either cemented or screw-retained. Porcelain crowns can only replace lost teeth, bland tissues and alveolar bone $(1,2,7)$. Fixed-detachable prosthesis, as original designed by Dr. Bra-nemark, also known as hybrid prosthesis. It presents an inner overcast made of titanium, a precious alloy or zirconium, on which the acrylic gingival or teeth, also acrylic, are placed. There is a variation to this design where porcelain or gingival composite is used with all the ceramic crowns cemented to the overcast $(1-3,7,8)$. 
Implant mucous-retained overdentures. They are a simple and efficient solution for patients with whole prosthesic stability problems. The function of the implant is only that of retaining on the mucous rest. Two implants at least and never more than four are necessary for their making.

Fewer implants than the totally implant supported overdenture are necessary, and the procedure of their making is simple and cheap. They have O-ring, locator and round bars with Hader clips, among others, kinds of attachments, which anchor the implant to the overdenture. It is a resilient anchorage system which allows vertical and rotary movement of the overdenture. This prosthesic design uses bone crowns as primary holding areas. Chronic occlusion pressure would inevitably produce a progressive wearing off the alveolar bone, except for the areas where implants are placed $(1,9)$.

Implant supported overdentures. Milled bar and telescopic overdentures are under this group.The implant acts as retainer and prosthesic holder. They present a rigid anchorage system between the implant and the overdenture, and do not need to be held on soft tissues. Its operational biomechanism is similar to that of fixed porcelain and hybrid prostheses. The implant supported milled bar overdenture presents one or two milled bars that are conically oriented from 2 to 10 grades. The overdenture, which is removable, is precisely and rigidly adjusted to the bar, limiting its lateral and rotary movements. Its rigid anchorage system evenly distributes stresses caused by the different forces along the implant complex. Besides, this overdenture includes fastenings which minimize the overdenture movement along its path of insertion. (1-5, 7-10). Fixed porcelain prostheses are recommended in clinical cases where soft tissues and alveolar bones are intact-only lost teeth are replaced-. Hybrid prostheses and implant supported milled bars overdentures make it easier to obtain better aesthetic results in clinical cases where there have been losses of hard and soft tissues $(2,4,7)$.

\section{Material and Methods}

This bibliographical review searched indexed articles dealing with relevant aspects of the mille bar overdenture published on Medline between 1998-2008. This search included Pub Med, Science Direct, Wiley InterScience, Embase, The Cochrane and Scielo. A total number of 20 articles fulfilled the necessary requirements to be included in this study.The key words used are milled bar, dental implants, dental prosthesis, implant-supported Overdentures.

\section{Results}

Success rates of the implant-supported milled bar overdenture and complication effects.

The implant-supported prosthesic restoration offers excellent rates of success in mandible and upper jaw ca-ses, by applying either direct or indirect protocol forces $(2,3,5,6,11)$.Implant-supported mandibular overdentures present higher complication and failure rates in upper maxilla cases as compared to mandible cases, especially when they are used as a rescue or emergency treatment in patients originally planned for fixed prostheses $(9,10,12,13)$.It is worth mentioning the studies carried out by Krenmair et al., showing a cumulative survival rate of implants on the milled bar overdenture after 5 years of $99 \%$ in the mandible and of $97.8 \%$ in the upper maxilla $(8,10)$. The milled bar implant-supported overdenture presents lower prosthesic complication rates and need lesser maintenance service as compared to implant-retained prostheses, which use a resilient anchorage system $(5,8,10)$.

Number and position of implants.

Nowhere in the international literature can we find detailed protocols on the minimum number of implants and the necessary characteristics to hold an overdenture retained with a milled bar. More studies on the issue are necessary to get to deeper conclusions $(1,12)$. Nevertheless, there is a tendency to place at least 4 implants between the mentonian holes and 4-6 implants in the upper maxilla $(1,2,7,9,14,15)$.

Milled bar design.

Milled bars are directly screwed either on implants or the transgingival abutments. Depending on how high the bar is over the soft tissue, the bars are milled 2-10 grades conically oriented. The higher the more conically oriented. When the implants are placed on the anterior area of the first premolar teeth, the bar presents cantilevers towards the distal teeth which improve overdenture stability. The extension of the cantilevers will never overcome the anterior-posterior distance between the middle and distal implant by 1.5 times $(2,4,8,10)$.

Types of metal used in the making of milled bars.

We can use to cast the bars precious metal alloys, nonprecious metals such as cr- co or titanium $(9,10,14)$.

Materials used in the making of the overdenture which is fixed on the milled bar.

The overdenture presents an inner metal or acrylic suprastructure which adjusts precisely and rigidly to the milled bar $(1-4,9,14)$.

Techniques used in the making of milled bar overdentures.

Different techniques are nowadays used in the making of milled bar overdentures. By using electro formation machinery, the CAD/CAM technology or the fine traditional techniques also used to make removable prostheses $(1,3,14)$.

Type and number of attachments.

There is a great variety of designs the practitioner may use as he/she finds suitable. The type andnumber of attachments used, their location in the bar and their mor- 
phology (conicity, retention capacity of the suprastructure, length of the projections, etc.), provides the overdenture set with a biomechanical behaviour similar to that of fixed-prosthetic implant restoration. The specific function of the attachments on implant-supported milled bar overdentures is simply that of fixing the prosthesis, limiting its movements along its path of insertion $(1,3,5$, 8-10, 14). Advantages of implant supported milled bar overdentures over fixed ceramometal prostheses used in moderate to severe reabsorbed maxilla clinical cases. When there is a loss of soft and hard tissues, especially in the upper maxilla, fixed porcelain prostheses present different problems: the correct necessary position of the implants to obtain the right aesthetics may require complex surgical reconstruction of soft and hard tissues. The big size of the prosthesic restoration may make prophylaxis difficult and increase costs. The implantsupported milled bar overdenture is a much cheaper alternative, offers a retention and a stability similar to those of fixed-prosthesic implant restorations, and the right position of the implants to obtain the ideal aesthetics is not such critical as it is the case with fixed porcelain prostheses. This easiness to get the right aesthetics makes surgery easier and avoids tissue generating processes. The overdenture is removable, with simple oral hygiene and the possibility to remove it at night is an efficient way to solve bruxism problems $(1,7,9,16)$. Advantages of milled bar implant-supported overdentures over hybrid prostheses in maxilla. Hybrid fixed-remova-ble prostheses, as originally designed by Dr. Branemark, can present a series of problems for the maxilla whenever there is an important alveolar reabsorbtion. The extended labial flanges of the prosthesis necessary to provide the correct lip support occasionally create areas of difficult hygiene care, where food as well as plaque can be accumulated. The diminishing of these labial flanges in order to improve hygiene care could, on patience's feeling of air and saliva escaping. Also, clinical cases where implant screw-accesses open up the buccal surface involve important aesthetic problems for the screwed prostheses. Milled bar overdentures are the ideal alternative in such clinical situations $(1,3,9)$. Advantages of the implant-supported milled bar overdenture over the fixed-prosthetic implant restoration in clinical cases of moderate to severe reabsorbed edentulous ridges. Implant-supported milled bar overdentures are completely implant-supported and need no use of the edentulous ridges as primary stress-bearing areas, avoiding progressive bone loss caused by occlusion stress. In upper maxilla cases the design lay the soft palate bare, increasing the comfort of the patient. Its biomechanical performance, similar to that of fixed prostheses, makes them suitable for patients who fell that the retention and stability provided by implant mucous retained overdentures are not enough $(5,7,10,14,17)$. The implant supported milled bar overdenture is an efficient treatment for patients who have either suffered the surgical ablation of maxillary tumours or present genetic problems which cause lack of some teeth and the underdevelopment of dental crowns. In such cases, implantretained overdentures may present too many stability, retention, chewing and speech problems $(15,17,18)$.

\section{Discussion}

The implant supported milled bar overdenture is a therapeutic option requiring its correct diagnosis and adequate therapeutic planning from start. When it is well planned, it offers excellent long run rates of success in the upper maxilla and mandible. Clinic cases originall designed for fixed prosthetic implant restoration and later reconstructed with overdentures present higher failure rates, especially in the upper maxilla. In such cases, the overdenture is a rescue or an emergency treatment (8-10). Limiting factors such as lower bone quality (type 3 or 4, according to Lekholm and Zarb's classification), pumping up of maxilla sinuses, presence of the nasal fossae, the centripetal alveolar reabsorption, etc., are frequent in upper maxilla cases where there is a severe to moderate bone wearing off. All of these factors are related to a higher failure rate of the upper maxilla overdenture over the mandible overdenture $(12,9,20)$. Compared to the implant mucous supported overdenture, the implant supported milled bar overdenture presents neither a mucous rest or movement, limiting bone reabsorption of the crowns and extending the life of their attachments (due to their lesser use), decreasing prosthetic complications and maintenance needs (8-10). The minimum number of implants needed in milled bar overdentures, their placement and characteristics, are concepts still to be decided on in the international literature. They should be established for each individual case, taking into account that the mechanism of these prostheses is similar to that of fixed prostheses and that, if implants are only placed in the anterior area, distal cantilevers will be necessary to provide an adequate rear support $(1,7,12)$. The suprastructure is recommended to be precisely and rigidly adjusted to the milled bar and made of the same alloy. Otherwise, the wearing away of the area between the suprastructure and the milled bar can be accelerated, and the presence of different metals give rise to galvanism phenomena (4).Clinical and laboratory processes involved in the making of the implant supported milled bar overdenture are similar to those needed to make the hybrid fixed removable overdenture, as originally designed by Dr. Branemark. The fine techniques used with traditional removable overdentures allow the making of this type of prostheses without having to resort to expensive machinery, offering high quality at a low cost, as compared to CAD/CAM or spark-erosionsystems $(1,3,14)$. Depending on the de- 
sign of the prosthesis, the type, number and location of the attachments can vary. These attachments should fulfil the following characteristics: offer retention capacity, be cheap, need low maintenance cost, have the right dimensions, be easy to be replaced and allow an easy insertion and removal of the prosthesis (1-3, 8-10, 14). In moderate to severe reabsorbed edentulous ridge cases, the implant-supported milled bar overdenture is a therapeutic option which offers many advantages over the metal porcelain fixed or the hybrid fixed-detachable prostheses. Implant-supported milled bar overdentures present a similar stability and retention to that of the fixed prosthesis, with the advantages of the removable ones. The labial flanges can be extended as needed, making hygiene care easier and providing the right lip support. They can also be extended in clinical edentulous ridge cases which are visible when laughing, avoiding the transitional area between the gingival acrylic resin and the patient's mucous from being shown. The emerging of screws fixing the bar to implants in its vestibular side does not affect the aesthetics of the overdenture (as it happens with screwed prostheses) and the wrong position of some of the implants is not such a serious problem. Besides, the prosthesis can be removed at night to solve bruxism problems $(1,2,4,7,9,16)$.

Implant supported milled bar overdentures make it easier to replace the lack of tissues and to provide hygiene care in patients who have suffered the surgical ablation of maxillary tumours or present genetic problems causing lack of one or several teeth or the underdevelopment of dental crowns. Their high level of retention and stability improve patients' comfort, and their scarce mucous rest avoid trauma of soft tissues, avoiding possible future problems of osteorradionecrosis in irradiated patients $(17,18)$.

\section{Conclusions}

The implant supported milled bar overdenture is a very interesting treatment option for patients with moderate to severe reabsorbed maxilla problems. They offer the advantages of removable prostheses with the stability and retention of fixed prostheses.

\section{References}

1. Galindo DF. The implant-supported milled-bar mandibular overdenture. J Prosthodont. 2001;10:46-51.

2. Hebel KS, Galindo D, Gajjar RC. Implant position record and implant position cast: minimizing errors, procedures and patient visits in the fabrication of the milled-bar prosthesis. J Prosthet Dent. 2000;83:107-16.

3. Ercoli C, Graser GN, Tallents RH, Hagan ME. Alternative procedure for making a metal suprastructure in a milled bar implantsupported overdenture. J Prosthet Dent. 1998;80:253-8.

4. Finley JM. Restoring the edentulous maxilla using an implantsupported, matrix-assisted secondary casting. J Prosthodont. 1998;7:35-9.

5. Dudic A, Mericske-Stern R. Retention mechanisms and prosthetic complications of implant-supported mandibular overdentures: longterm results. Clin Implant Dent Relat Res. 2002;4:212-9.

6. Tolstunov L. Management of biomechanical complication of implant-supported restoration of a patient with combination syndrome: a case report. J Oral Maxillofac Surg. 2009;67:178-88.

7. Bedrossian E, Sullivan RM, Fortin Y, Malo P, Indresano T. Fixedprosthetic implant restoration of the edentulous maxilla: a systematic pretreatment evaluation method. J Oral Maxillofac Surg. 2008;66:112-22.

8. Krennmair G, Krainhöfner M, Piehslinger E. Implant-supported mandibular overdentures retained with a milled bar: a retrospective study. Int J Oral Maxillofac Implants. 2007;22:987-94.

9. Tipton PA. The milled bar-retained removable bridge implant-supported prosthesis: a treatment alternative for the edentulous maxilla. J Esthet Restor Dent. 2002;14:208-16.

10. Krennmair G, Krainhöfner M, Piehslinger E. Implant-supported maxillary overdentures retained with milled bars: maxillary anterior versus maxillary posterior concept--a retrospective study. Int J Oral Maxillofac Implants. 2008;23:343-52.

11. Uribe R, Peñarrocha M, Balaguer J, Fulgueiras N. Immediate loading in oral implants. Present situation. Med Oral Patol Oral Cir Bucal. 2005;10 Suppl 2:E143-53.

12. Sadowsky SJ. Treatment considerations for maxillary implant overdentures: a systematic review. J Prosthet Dent. 2007;97:340-8.

13. Goodacre CJ, Bernal G, Rungcharassaeng K, Kan JY. Clinical complications with implants and implant prostheses. J Prosthet Dent. 2003;90:121-32.

14. Brudvik JS, Chigurupati K. The milled implant bar: an alternative to spark erosion. J Can Dent Assoc. 2002;68:485-8.

15. Schoen PJ, Raghoebar GM, Bouma J, Reintsema H, Burlage FR, Roodenburg JL, et al. Prosthodontic rehabilitation of oral function in head-neck cancer patients with dental implants placed simultaneously during ablative tumour surgery: an assessment of treatment outcomes and quality of life. Int J Oral Maxillofac Surg. 2008;37:8-16. 16. Straioto FG, De Azevedo AM, Do Prado CJ, Das Neves FD, Neto AJ. Rehabilitation of maxillary edentulism with implant-supported milled-bar prostheses. Implant Dent. 2006;15:366-71.

17. Fukuda M, Takahashi T, Nagai H, Iino M. Implant-supported edentulous maxillary obturators with milled bar attachments after maxillectomy. J Oral Maxillofac Surg. 2004;62:799-805.

18. Takahashi T, Fukuda M, Funaki K, Tanaka K. Magnet-retained facial prosthesis combined with an implant-supported edentulous maxillary obturator: a case report. Int J Oral Maxillofac Implants. 2006;21:805-7.

19. Sorní M, Guarinós J, García O, Peñarrocha M. Implant rehabilitation of the atrophic upper jaw: a review of the literature since 1999. Med Oral Patol Oral Cir Bucal. 2005;10 Suppl 1:E45-56.

20. Sorní M, Guarinos J, Peñarrocha M. Implants in anatomical buttresses of the upper jaw. Med Oral Patol Oral Cir Bucal. 2005;10:163-8. 